\title{
PINCH expression in relation to radiation response in co-cultured colon cancer cells and in rectal cancer patients
}

\author{
ANNICA HOLMQVIST ${ }^{1,2}$, BIRGITTA HOLMLUND ${ }^{1,2}$, MALIN ARDSBY $^{1}$, \\ SURAJIT PATHAK ${ }^{1}$ and XIAO-FENG SUN ${ }^{1}$ \\ ${ }^{1}$ Department of Clinical and Experimental Medicine, Linköping University; ${ }^{2}$ Department of Medical Oncology, \\ County Council of Östergötland, Linköping University, Linköping, Sweden
}

Received May 20, 2013; Accepted July 15, 2013

DOI: $10.3892 /$ or.2013.2673

\begin{abstract}
Particularly interesting new cysteine-histidine rich protein $(\mathrm{PINCH})$, involved in cell spreading, motility and proliferation, has been shown to enhance radioresistance in colon cancer cell lines. The expression of PINCH in relation to radiation was studied in co-cultured colon cancer cells. Furthermore, the clinical significance between PINCH and radiotherapy (RT) was analyzed in rectal cancer patients with or without RT. The relative PINCH expression in colon cancer (KM12C) cells cultured separately and in co-culture was examined by western blotting and real-time PCR, and was analyzed over a period of 8 and $24 \mathrm{~h}$ after radiation. PINCH expression was immunohistochemically examined in 137 primary rectal tumors for which 65 cases did not receive RT and 72 cases received RT. PINCH expression tended to decrease from that in the separately cultured KM12C cells without radiation to that in cells with radiation at $8 \mathrm{~h}$ $(\mathrm{P}=0.060)$; while in the co-cultured cells, no significant difference was found $(\mathrm{P}=0.446)$. In patients with $\mathrm{RT}$, strong PINCH expression was related to worse survival, when compared to patients with weak expression, independent of TNM stage, degree of differentiation, age and p53 status ( $\mathrm{P}=0.029$, RR 4.03, 95\% CI 1.34-12.1). No survival relationship for the patients without RT was observed $(\mathrm{P}=0.287)$. A statistical interaction analysis between PINCH, RT and survival showed a trend towards significance $(\mathrm{P}=0.057)$. In conclusion, $\mathrm{PINCH}$ predicts survival in rectal cancer patients with RT, but not in patients without RT. The expression of PINCH may be regulated by radiation and by environmental factors surrounding the cells.
\end{abstract}

Correspondence to: Dr Annica Holmqvist, Department of Medical Oncology, County Council of Östergötland, Linköping University, S-58185 Linköping, Sweden

E-mail: annica.holmqvist@lio.se

Key words: co-culture, immunohistochemistry, $\mathrm{PINCH}$, prognosis, radiation, rectal cancer

\section{Introduction}

Particularly interesting new cysteine-histidine rich protein $(\mathrm{PINCH})$ is an adapter protein which forms a complex with integrin-linked-kinase (ILK) and parvin and connects integrins at the cell surface with the actin cytoskeleton of the cell. PINCH participates in the protein-protein interaction with downstream effectors that regulate cell shape, motility and survival (1-5), and PINCH has been shown to be related to worse survival in colorectal cancer, when expressed in tumor tissues, notably at the invasive edges $(6,7)$.

Few studies have analyzed the relationship between PINCH and radiotherapy (RT). Eke et al (8) demonstrated, in a cell line study, that PINCH was related to enhanced radioresistance. Our recent study involving rectal cancer patients performed on the same material as used in the present study showed that strong PINCH expression was related to worse survival when compared to weak expression in patients without RT, but not in patients with RT. A further statistical interaction analysis between PINCH, RT and survival showed no significant result, suggesting that RT was not directly the reason for the differences in survival between patients with weak and strong PINCH expression. In addition, no differences were found when the expression of PINCH was compared between unirradiated and radiated fibroblasts (7).

In the present study, we further investigated the relationship between PINCH and radiation in colon cancer cells separately cultured and in co-culture. The colon cancer cells were co-cultured as an attempt to imitate the tumor environment inside the body.

$\mathrm{C}$-Myc is a well-known regulator in the development of cancer, and controls key functions, such as cell proliferation, differentiation and apoptosis (9). Previously, it was shown in cell culture that PINCH was related to ERK, and ERK is a well-known regulator of C-Myc (10). Since both PINCH and $\mathrm{C}-\mathrm{Myc}$ have been shown to be involved in the regulation of cell proliferation and apoptosis, we wanted to further investigate the association between PINCH and C-Myc $(9,10)$.

The aim of the present study was to analyze the expression of PINCH in relation to radiation in separately and co-cultured colon cancer cells and to study the association between PINCH and C-Myc. Furthermore, the relationship between PINCH and preoperative RT was investigated in rectal cancer patients. 


\section{Materials and methods}

Cell lines. The colon cancer cell lines, KM12C, KM12SM and KM12L4a, were a kind gift from Professor I.J. Fidler (Anderson Cancer Center, University of Texas, TX, USA). $\mathrm{KM} 12 \mathrm{C}$ is derived from a patient with stage II colon cancer, KM12SM is a spontaneous liver metastasis (11) and KM12L4a is an experimental liver metastasis (12). Western blot analysis showed no differences in PINCH expression among the KM12C, KM12SM and KM12L4a cell lines. The KM12C cell line was chosen for further culturing proceedings as an attempt to imitate the tumor environment inside the body.

The separately cultured KM12C cells, and the co-cultured KM12C and CCD-18-Co cells were cultured in Eagle's minimal essential medium (MEM), and supplemented with $1 \%$ penicillin-streptomycin and 10\% FBS (ATCC; Rockville, MD, USA). The co-cultured cells were grown in a 6-well-plate with an insert chamber (pore size $0.4 \mu \mathrm{m}$ ).

Patients. Patients with rectal adenocarcinoma from the Southeast Swedish Health Care region who participated in a Swedish clinical trial of preoperative RT during 1987-1990 were included (13). Of the 137 primary tumors, 72 patients underwent surgery alone and 65 patients underwent preoperative RT prior to surgery. RT was delivered at 25 Gray (Gy) in 5 fractions during a median of 6 days (range, 5-12 days). Surgery was then performed a median of 3 days (range, 1-13 days) after RT. None of the patients received adjuvant chemotherapy before or after surgery. The mean age of the patients was 67 years (range, 36-85 years) and the mean follow-up was 80 months (range, 0-193 months). Additional characteristics of the patients and tumors are presented in Table I. The Research Ethics Committee of Linköping University Hospital, no. 86151, approved the study.

Radiation procedure. For all experiments, cells where seeded at a density of 60,000 cells $/ \mathrm{cm}^{2}$ and irradiated with photons from a $6 \mathrm{MV}$ linear accelerator (Varian Clinac $600 \mathrm{C} / \mathrm{D}$; Varian Medical Systems, Palo Alto, CA, USA). The cells were exposed to single doses of $0,2,5$ or $10 \mathrm{~Gy}$, respectively, at room temperature. A dose of $2 \mathrm{~Gy}$ was used for further analyses since this is the most commonly used dose in the clinic. The controls $(0 \mathrm{~Gy})$ were handled under the same environmental conditions as the treated cells. Following radiation, cells were harvested at 8 and $24 \mathrm{~h}$.

Western blot analysis. After radiation, cells were washed in PBS and lysed in RIPA buffer, containing $150 \mathrm{mM} \mathrm{NaCl} 2 \%$ Triton, $0.1 \%$ SDS, $50 \mathrm{mM}$ Tris pH 8.0 and a Protease Inhibitor Cocktail without chelating reagents (Sigma-Aldrich, Stockholm, Sweden). The protein concentration was determined using the colorimetric BCA protein assay reagent (Pierce, Woburn, MA, USA). Samples containing $30 \mu \mathrm{g}$ protein were separated by electrophoresis on a Mini-PROTEAN TGX ${ }^{\mathrm{TM}}$ precast $12 \%$ gel (Bio-Rad, Hercules, CA, USA) for $55 \mathrm{~min}$ at $200 \mathrm{~V}$. The separated proteins were transferred to a PVDF membrane $(0.45 \mu \mathrm{m}$; polyvinylidene fluoride transfer membrane; VWR/ Life Sciences, Pall Corp., Pensacola, FL, USA). The membranes were blocked with $5 \%$ non-fat dried milk in Tris-buffered saline (TBS) containing 0.1\% Tween-20 (TBST) and incubated
Table I. Patient and tumor characteristics.

\begin{tabular}{|c|c|c|c|}
\hline Characteristics & $\begin{array}{l}\text { No radiotherapy } \\
\qquad \begin{array}{c}\mathrm{n}=72 \\
\mathrm{n}(\%)\end{array}\end{array}$ & $\begin{array}{l}\text { Radiotherapy } \\
\qquad \begin{array}{c}\mathrm{n}=65 \\
\mathrm{n}(\%)\end{array}\end{array}$ & $\mathrm{P}$-value \\
\hline Gender & & & 0.702 \\
\hline Male & $42(58)$ & $40(62)$ & \\
\hline Female & $30(42)$ & $25(38)$ & \\
\hline Age (years) & & & 0.842 \\
\hline$\leq 67$ & $30(42)$ & $26(42)$ & \\
\hline$>67$ & $42(58)$ & $39(60)$ & \\
\hline TNM & & & 0.105 \\
\hline I & $20(28)$ & $22(34)$ & \\
\hline IIA & $18(25)$ & $21(32)$ & \\
\hline IIIA & $8(11)$ & 1 (2) & \\
\hline IIIB & $11(15)$ & $11(17)$ & \\
\hline IIIC & $11(15)$ & $4 \quad(6)$ & \\
\hline IV & $4(6)$ & $6 \quad(9)$ & \\
\hline Differentiation & & & 0.263 \\
\hline Well & $2(3)$ & $2(3)$ & \\
\hline Moderate & $58(81)$ & $48(74)$ & \\
\hline Poor & $12(16)$ & $15(23)$ & \\
\hline Local recurrence & & & 0.059 \\
\hline No & $57(79)$ & $59(91)$ & \\
\hline Yes & $15(21)$ & $6(9)$ & \\
\hline Distant recurrence & & & 0.257 \\
\hline No & $42(58)$ & $44(68)$ & \\
\hline Yes & $30(42)$ & $21(32)$ & \\
\hline
\end{tabular}

with the primary PINCH antibody $(1 \mu \mathrm{g} / \mathrm{ml})$. The antibodies were incubated overnight at $4^{\circ} \mathrm{C}$ in TBST in $1 \%$ non-fat dried milk. The membranes were washed and incubated with an HRP-conjugated polyclonal goat anti-mouse secondary antibody (1:5000; DakoCytomation, Glostrup, Denmark) for $1 \mathrm{~h}$ at room temperature, followed by enhanced chemiluminescence (ECL) (Amersham Biosiences/GE Healthcare). To verify equal loading of the wells, the membranes for PINCH were re-incubated with a primary mouse polyclonal anti- $\beta$-actin antibody (1:5000; Sigma-Aldrich, Steinheim, Germany). All experiments were repeated three times.

RT-PCR. The relative abundances of PINCH and C-Myc mRNA were determined by real-time PCR (RT-PCR) with duplicates of each sample, repeated 3 times. Total RNA was extracted from KM12C and CCD-18-Co cells by using the RNA Blood Mini kit (Qiagen), and cDNA was transcribed using the High Capacity cDNA reverse transcription kit according to the manufacturer's instructions (Applied Biosystems). PINCH and C-Myc mRNA expression was determined by using specific primers Hs00757864_m1 for PINCH and Hs00905030_m1 for C-Myc (Applied Biosystems). The RT-PCR reactions were performed using the 7500HT Fast RT-PCR system, and the data were displayed graphically using the SDS 3.2 software 
A

KM12C

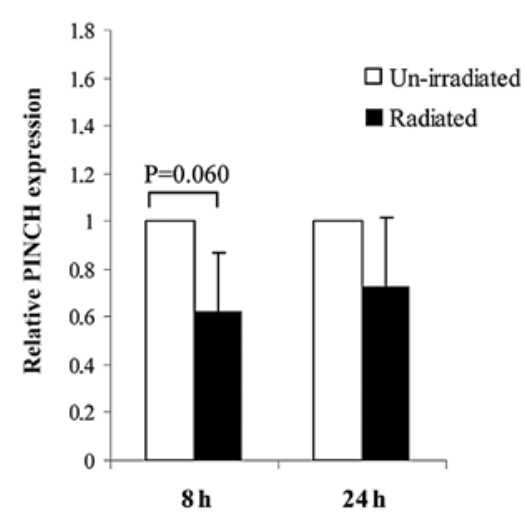

B KM12C Co-culture

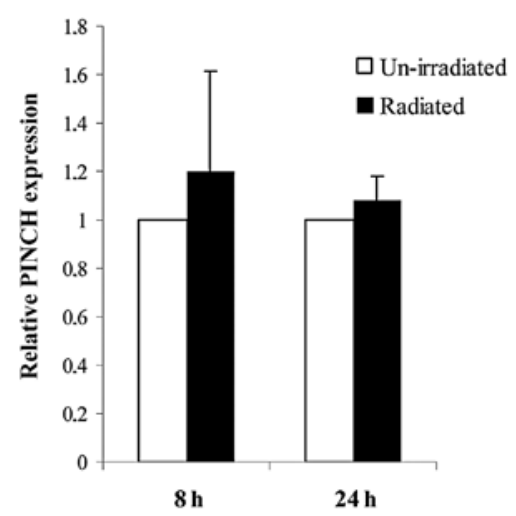

Figure 1. RT-PCR analysis of relative PINCH expression in (A) separately cultured and (B) co-cultured KM12C cells without or with RT analyzed at 8 and $24 \mathrm{~h}$ after radiation. The results are measured by densitometry and shown as the difference between radiated and control samples. The baseline (1.00) represents the control levels.

program (Applied Biosystem). The scores from the gene of interest and the mean scores of two reference genes, glyceraldehyde 3-phosphate dehydrogenase (GAPDH) and $\beta$-actin (Applied Biosystems), were used for further calculations by using the $\Delta \Delta \mathrm{Ct}$ method (14).

Protein-protein interaction analysis. A protein-protein interaction analysis was carried out to determine the possible interaction between PINCH and C-Myc. Amino acid sequences of human LIM and senescent cell antigen-like-containing domain (LIMS1) (Uniprot ID, P48059) (PINCH) and human Myc proto-oncogene protein (Myc) (Uniprot ID, P01106) were downloaded from UniProt. Subsequently, sequences were submitted to I-TASSER server. C-score was preferably within the range of 2 to -1.5 . A further protein interaction analysis by using the PIPS software method was used, where four different features were considered; expression data, orthology interaction relationships, protein features and network topology. The scores calculated by each of these features were combined to output the final interaction score.

Immunohistochemistry. Formalin-fixed paraffin-embedded sections were deparaffinized in xylene and rehydrated with a graded series of ethanol. The sections were treated by high pres- sure cooking for $10 \mathrm{~min}$ with Tris-ethylenediaminetetraacetic acid (EDTA) buffer ( $\mathrm{pH} \mathrm{9.0)} \mathrm{and} \mathrm{stored} \mathrm{at} \mathrm{room} \mathrm{temperature}$ for $30 \mathrm{~min}$. Following pre-incubation in methanol with $0.3 \%$ $\mathrm{H}_{2} \mathrm{O}_{2}$ for $20 \mathrm{~min}$, the sections were incubated with Dako Protein Block (Dako, Carpinteria, CA, USA) for $10 \mathrm{~min}$ and further incubated with rabbit anti-PINCH antibody at $6 \mu \mathrm{g} /$ $\mathrm{ml}$ in antibody diluent (Dako) for $1 \mathrm{~h}$ at room temperature. After washing in phosphate-buffered saline (PBS, pH 7.4), the sections were incubated with a mouse anti-rabbit secondary antibody provided in the Dako ChemMate EnVision detection kit (Dako) at room temperature for $25 \mathrm{~min}$ and then washed with PBS. The sections were further subjected to 3,3'-diaminobenzidine tetrahydrochloride for $8 \mathrm{~min}$ and counterstained with hematoxylin. The positive controls were primary colorectal tumors known to stain positive for PINCH, and the negative controls were primary rectal tumors where PBS was used instead of the primary antibody. In all staining procedures, the positive control showed clear staining, and no staining was observed in the negative controls.

The results of PINCH expression in tumors consisted of the scores determined by two independent authors (7) in a blinded fashion without any knowledge of the clinical and biological information. The percentage of stained cells was estimated among the total number of cells by reading 10-20 areas at a magnification of $x 400$, regardless of the staining intensity. The cases were scored as $<25 \%, 25-49 \%, 50-74 \%$ or $\geq 75 \%$, respectively. To avoid an artificial effect, the cells on the margins of sections and areas with poorly presented morphology were not counted. The results of the staining intensity was presented in our previous study (7) and will therefore not be further discussed in this study.

Statistical analysis. For the KM12C cell results analyzed by western blotting and RT-PCR, an independent t-test by group was used to evaluate whether there were any significant differences between radiated and unirradiated cells in the expression of PINCH and C-Myc separately cultured or in co-culture. For the patients, the Chi-square method was used to analyze the relationship between PINCH expression in tumors and the clinical or pathological factors. Cox's proportional hazard model was used to estimate the relationship between PINCH expression and survival, including both univariate and multivariate analyses. Survival curves were computed according to the Kaplan-Meier method. For all statistical analysis including the interaction analysis between PINCH, RT and survival the statistical software program, STATISTICA, was used. Tests were two-sided, and $\mathrm{P}<0.05$ was considered to indicate a statistically significant result.

\section{Results}

PINCH expression in KM12C and CCD-18-Co cells cultured separately or co-cultured and treated with radiation or without. The expression of PINCH in KM12C cells cultured separately and in co-culture by RT-PCR was compared between unirradiated and radiated cells at 8 and $24 \mathrm{~h}$. PINCH expression tended to decrease from separately cultured $\mathrm{KM} 12 \mathrm{C}$ cells without radiation to cells with radiation at $8 \mathrm{~h}$ $(\mathrm{P}=0.060)$, but not at $24 \mathrm{~h}$ (Fig. 1A). In co-cultured KM12C cells, no significant differences in PINCH expression were 
$\mathbf{A}$

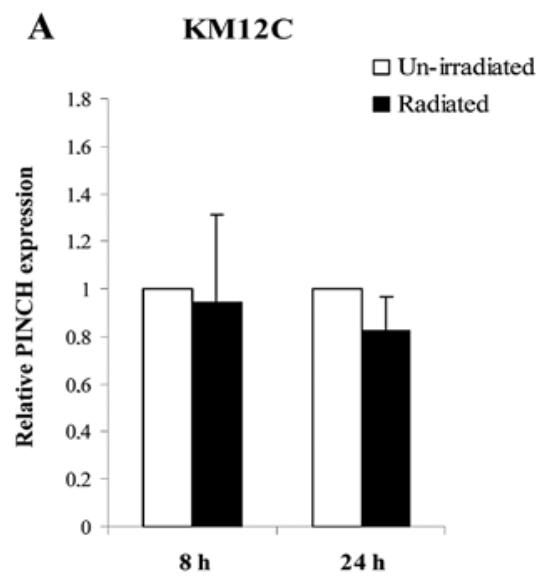

B KM12C Co-culture

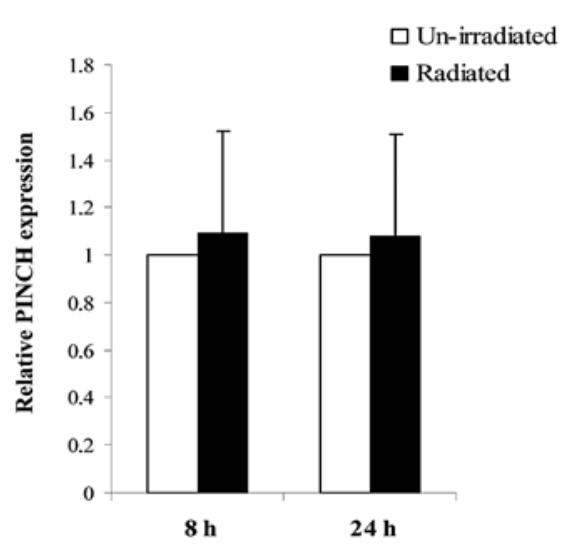

C KM12C

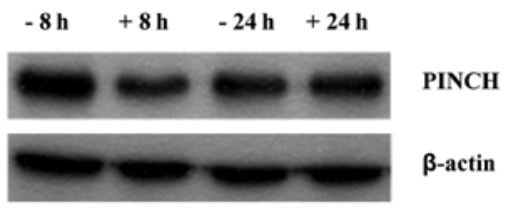

D KM12C Co-culture

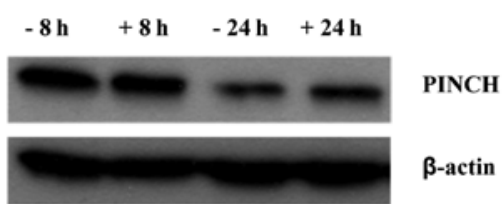

Figure 2. Western blot analysis of the PINCH expression in separately and co-cultured KM12C cells without (-) or with RT (+) analyzed at 8 and $24 \mathrm{~h}$ after radiation. Densitometric measurement $(\mathrm{A}$ and $\mathrm{B})$ of the samples (C and D) are shown as the difference between radiated and control samples. The baseline (1.00) represents the control levels.

found between unirradiated and irradiated cells at 8 and $24 \mathrm{~h}$ (Fig. 1B). A similar, but weaker expression pattern, compared to RT-PCR was found when the expression of PINCH was analyzed by western blotting in unirradiated and irradiated KM12C cells cultured separately and in co-culture at 8 and $24 \mathrm{~h}$, but no significant difference was achieved (Fig. 2A-D).

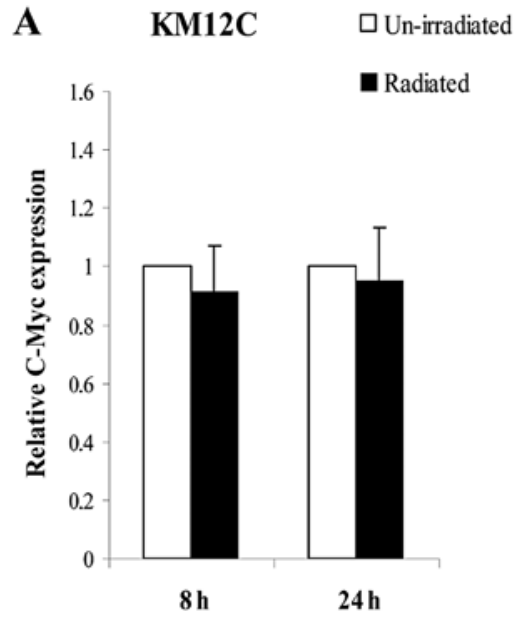

B KM12C Co-culture $\square$ Un-iradiated

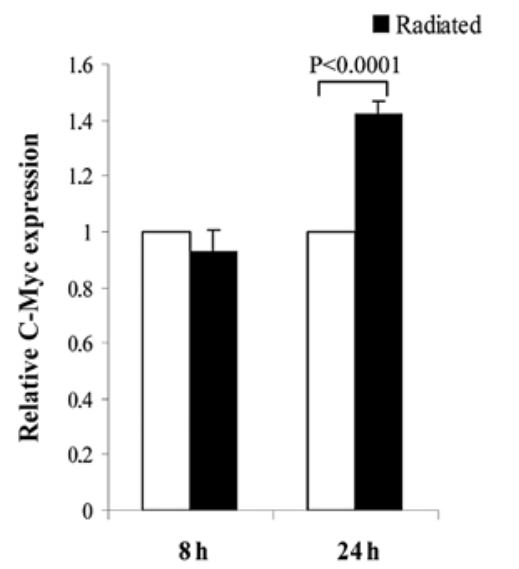

Figure 3. RT-PCR analysis of C-Myc expression in (A) separately cultured and (B) co-cultured KM12C cells without or with RT analyzed at 8 and $24 \mathrm{~h}$ after radiation. The difference between radiated and unirradiated results was measured by densitometry. The baseline (1.00) represents the control levels.

C-Myc expression in KM12C and CCD-18-Co cells cultured separately or in co-culture and treated with radiation or without. The C-Myc expression as analyzed by RT-PCR in separately and co-cultured KM12C cells was further compared between unirradiated and irradiated cells at 8 and $24 \mathrm{~h}$. In the separately cultured KM12C cells with radiation, compared to the cells without radiation, $\mathrm{C}-\mathrm{Myc}$ was equally expressed at 8 and 24 h (Fig. 3A), while in co-cultured KM12C cells with radiation, C-Myc showed an equivalent expression at $8 \mathrm{~h}$ and a significantly increased expression at $24 \mathrm{~h}$ when compared to the cells without radiation $(\mathrm{P}<0.0001$, Fig. 3B).

Interaction analysis between human PINCH, senescent cell antigen-like-containing domain (LIMS1) and human Myc proto-oncogene protein $(C-M y c)$. The interaction between human LIM and senescent cell antigen-like-containing domain (LIMS1) (PINCH) (Uniprot ID, P48059) and human Myc proto-oncogene protein (C-Myc) (Uniprot ID, P01106) was further studied. To model the protein structure, the sequences of both the proteins were PSI-BLASTed against the PDB proteins. Due to the unavailability of appropriate template with significant identity and query coverage, comparative modeling was not able to be performed. Next the 

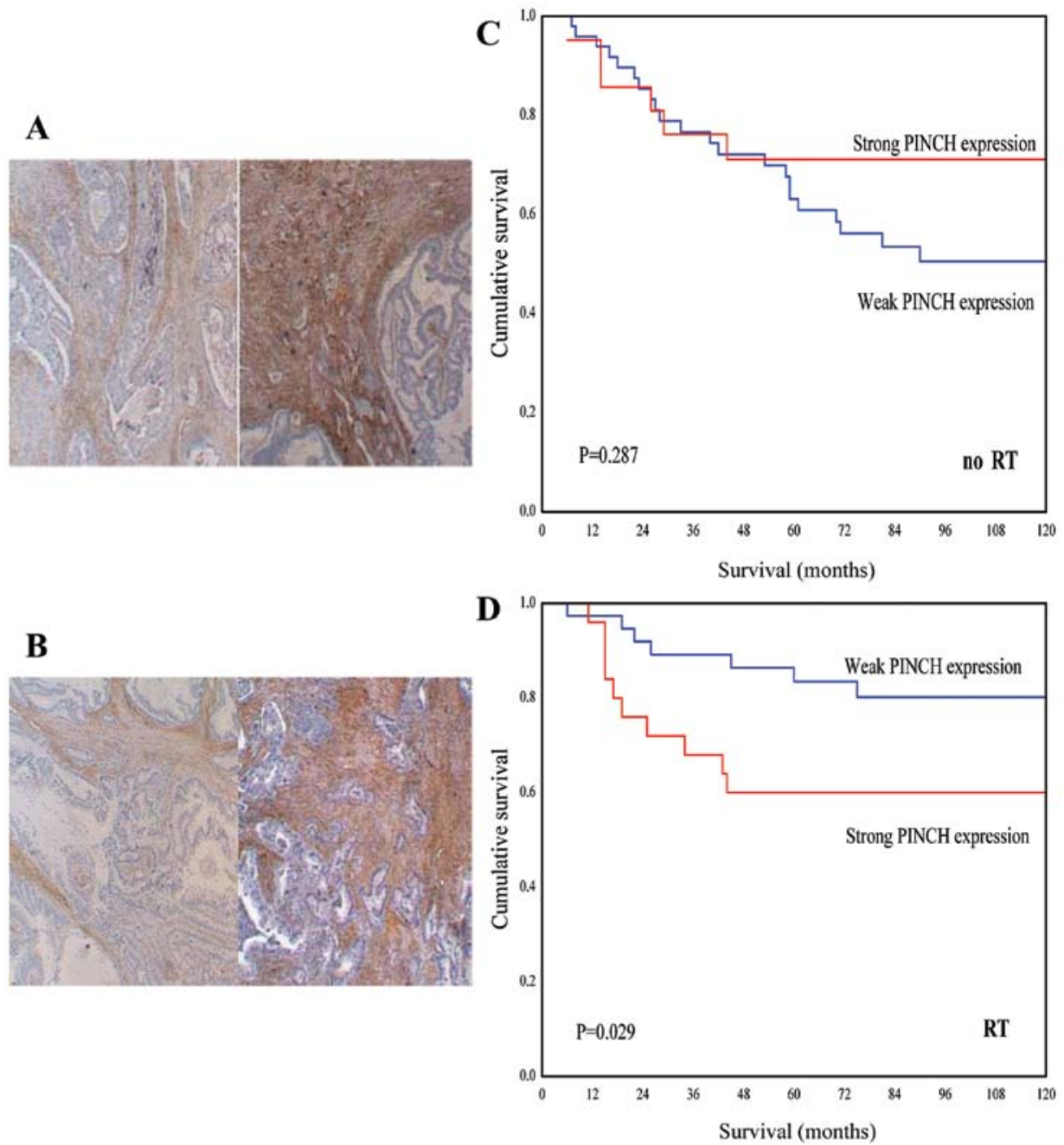

Figure 4. Weak and strong PINCH expression in rectal tumors (A) without or (B) with RT. PINCH expression in relation to overall survival in rectal cancer patients (C) without or (D) with RT.

same fold recognition method as the I-TASSER server was tested (Table II), however, it also reported structures having $50 \%$ loop region and $>50 \%$ of the model lacked proper secondary structures. The secondary structures were counter checked by predicting all available isoforms of LIMS1 and C-Myc which confirmed the I-TASSER results. A further protein interaction analysis using the PIPS software showed an interaction score of 0.052 , and no green domains were found when the Chi square scores for co-occurrence of domains were studied.

PINCH expression in primary rectal tumors with or without $R T$. By analyzing the entire tumor area, the staining percentage of PINCH in the primary tumors without (Fig. 4A) or with RT (Fig. 4B) was evaluated. Of the 137 tumors, 9 cases (7\%) had $<25 \%$ staining, $17(12 \%)$ cases had $25-49 \%$ staining, 62 (45\%) cases had 50-74\% staining and 47 (34\%) cases had $\geq 75 \%$ PINCH staining. For further analysis, the cut-off point for the staining percentage of PINCH expression was set to $75 \%$. The cases with negative and $<74 \%$ stained cells were classified as the weak expression subgroup, and the cases with $\geq 75 \%$ stained cells were classified as the strongly PINCH expression subgroup.
Table II. Protein interaction analysis between LIMS1/PINCH and C-Myc.

\begin{tabular}{lccr}
\hline $\begin{array}{l}\text { Best model from } \\
\text { I-TASSER }\end{array}$ & C-Score $^{\mathrm{a}}$ & TM-Score $^{\mathrm{b}}$ & RMSD $^{\mathrm{c}}(\AA)$ \\
\hline LIMS1/PINCH $^{\mathrm{d}}$ & -3.77 & $0.31 \pm 0.10$ & $15.7 \pm 3.3$ \\
C-Myc & -1.18 & $0.57 \pm 0.15$ & $9.7 \pm 4.6$ \\
\hline
\end{tabular}

${ }^{\mathrm{a}} \mathrm{C}$-Score (Confidence score), which assesses the accuracy of the predicted structure based on the quality of the threading alignments, reports a score between -5 and 2 expected to be positive and at least $>-1.5$. ${ }^{\mathrm{b}} \mathrm{TM}$-Score (Template Modeling score) is defined to evaluate the topological similarity of protein structure pairs with a value in the range of $(0,1)$. Statistically, a TM-Score of $<0.17$ means a randomly selected protein pair with the gapless alignment taken from PDB; a TM-score of $>0.5$ corresponds to the protein pairs of similar fold. ${ }^{\mathrm{c}} \mathrm{RMSD}$, Root Mean Square Deviation. ${ }^{\mathrm{d}}$ LIMS1 or senescent cell antigen-like-containing domain is the gene symbol for particularly interesting new cysteine-histidine rich protein (PINCH).

We further analyzed the relationship between the staining percentage of PINCH and survival in patients without or with 
Table III. Multivariate analysis of PINCH expression and clinicopathological features in rectal cancer patients with radiotherapy.

\begin{tabular}{ccc}
\hline Features & $\begin{array}{c}\text { Patients, } \mathrm{n}=65 \\
\mathrm{n}(\%)\end{array}$ & Cancer death rate ratio (95\% CI)
\end{tabular}

\begin{tabular}{|c|c|c|c|}
\hline PINCH expression & & & 0.029 \\
\hline Weak & $39(60)$ & 1.0 & \\
\hline Strong & $26(40)$ & $4.03(1.34-12.1)$ & \\
\hline Age (years) & & & 0.521 \\
\hline$<67$ & $26(40)$ & 1.0 & \\
\hline$\geq 67$ & $39(60)$ & $0.73(0.21-2.56)$ & \\
\hline TNM stage & & & 0.0004 \\
\hline I & $22(34)$ & 1.0 & \\
\hline IIA & $21(32)$ & $3.47(0.37-32.8)$ & \\
\hline IIIA & $1 \quad(2)$ & $0^{\mathrm{a}}(0-32.8)$ & \\
\hline IIIB & $11(17)$ & $7.14(0.71-72.1)$ & \\
\hline IIIC & $4(6)$ & $32.3(2.74-378.9)$ & \\
\hline IV & $6 \quad(9)$ & $138.2(11.2-1701.2)$ & \\
\hline Differentiation degree & & & 0.503 \\
\hline Well & $5(8)$ & 1.0 & \\
\hline Moderate & $39(60)$ & $1.02(0.08-12.5)$ & \\
\hline Poor & $21(32)$ & $1.21(0.10-14.5)$ & \\
\hline p53 status & & & 0.204 \\
\hline Negative & $51(76)$ & 1.0 & \\
\hline Positive & $14(24)$ & $2.08(0.67-6.44)$ & \\
\hline
\end{tabular}

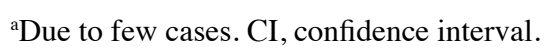

RT. In the patients with RT, strong PINCH expression tended to be related to worse survival when compared to the patients with weak PINCH expression $(\mathrm{P}=0.056)$. After adjusting for TNM stage, degree of differentiation grade, age and p53 status, the relationship reached statistically significance ( $\mathrm{P}=0.029$; RR, 4.03; 95\% CI, 1.34-12.1) (Fig. 4D, Table III). No statistically significant difference was found in the patients without RT ( $\mathrm{P}=0.287$, Fig. 4C). A further statistical interaction analysis between PINCH, RT and survival showed a trend towards significance $(\mathrm{P}=0.057)$. No significant difference in PINCH expression was found in the subgroups with no RT and with RT for disease-free survival, local recurrence-free survival and distant recurrence-free survival $(\mathrm{P}>0.5)$.

\section{Discussion}

In the present study, we demonstrated that the expression of PINCH tended to change when the separately cultured cancer cells were compared with the co-cultured cells. In separately cultured cancer cells at $8 \mathrm{~h}$, PINCH tended to decrease following RT when compared to cells without RT, while in co-cultured cancer cells there was no significant difference. It has been shown in cell culture that $\mathrm{PINCH}$ is regulated by EGF and PDGF via Nck-2 (15). The different expression pattern for PINCH in co-culture cells, compared to separately culture cells, may be caused by growth factors that regulate the expression of PINCH.
Even though we noted slight changes in the expression of PINCH between separately cultured and co-cultured cancer cells, we did not find in the present study, nor in our previous study (7) a high enough difference in PINCH expression between radiated and unirradiated cells to be able to conclude that the expression of PINCH was regulated by RT. Therefore, we re-analyzed the expression of PINCH using the same patient material that we used in our previous study (7). In the previous study, no significant results were found for the staining percentage of PINCH when the cut-off point for PINCH percentage was set to $50 \%$. In the present study, the cut-off point was set to $75 \%$. Moreover, in the present study, the PINCH staining of the entire tumor section was analyzed compared to the previous study where only the staining intensity at the invasive margin showed significant relationships (7). Noteworthy, when the entire tumor area using the same material as described was re-investigated for the staining percentage of $\mathrm{PINCH}$, we found that in the patients with RT, the survival of patients with strong PINCH expression was significantly decreased when compared with the patients with weak PINCH expression after adjustment for TNM stage, differentiation degree, age and p53 status. In the patients without RT, no statistically significant difference was found. An interaction analysis was further performed in order to investigate whether RT was the reason for the change in survival between patients with weak and strong PINCH expression. The interaction analysis showed a trend towards 
significance, which may indicate that RT was the reason for the survival differences in the patients with weak and strong PINCH expression. In our previous study, it was shown that strong PINCH expression was related to worse survival when compared to weak expression in the patients without RT, while in the patients with RT, the significant difference was lost (7). When the invasive margin of the tumor was investigated, we found a relationship between strong PINCH expression and reduced survival in the patients without RT (7) while in the present study, when the entire tumor area was investigated, the same relationship was found but in the patients with RT, suggesting that the relationship between PINCH, RT and survival may depend on where PINCH is located in the tumor and how the immunostaining for PINCH is evaluated (percentage or intensity).

Few studies have analyzed the relationship between PINCH and RT. A recent cell line study of mouse embryonic fibroblasts and human colon, lung, cervix, skin and pancreas tumors showed that PINCH enhances radioresistance by activating Akt (8) while others found $\mathrm{PINCH}$ radiosensitivity to be similar even when the cells were grown in a suspension or under adherent conditions (16). The significant differences in survival of the patients with weak and strong PINCH expression treated with RT, and the positive interaction analysis between PINCH, RT and survival support our theory that the expression of PINCH may be regulated by RT. An in-depth cell line study and a study using a larger sample of rectal cancer patient material are ongoing to further confirm this relationship.

We found significantly increased expression of C-Myc at $24 \mathrm{~h}$ in co-cultured cancer cells treated with radiation compared to cells without radiation, which was not found in the separately cultured cancer cells. Previously, it was shown in a cell culture of colonic adenoma cells that growth factors play an important role in the regulation of the production of C-Myc (17). Other studies have shown that RT upregulates the number of growth factor receptors in cancer cells $(18,19)$. The significant increase in C-Myc expression at $24 \mathrm{~h}$ in co-cultured cancer cells treated with radiation compared to cancer cells without radiation may be due to growth factors produced in co-culture but not in separately cultured cells that together with radiation, not at $8 \mathrm{~h}$ but at $24 \mathrm{~h}$ after radiation, activate receptors on the cell surface, which further increases the expression of C-Myc. Since C-Myc has been shown to reduce apoptosis $(20,21)$, we suggest that in co-culture compared to separately cultured cells, cancer cells may become resistant to radiation by upregulation of C-Myc.

We found almost the same but a weaker expression pattern for PINCH compared to C-Myc in separately and co-cultured cancer cells with or without radiation, suggesting that there may be some relationship between PINCH and C-Myc. To further study the interaction between PINCH and C-Myc, a DNA sequence analysis was performed. Due to the unavailability of appropriate secondary structures, we were unable to further study the interaction between PINCH and C-Myc by bioinformatics approach. However, a bioinformatics analysis depends on previously published studies concerning the protein structure. Thus, it is evident that studies concerning these proteins have not been previously reported. We conclude that the two proteins do not directly interact, but may influence the expression of each other depending on other proteins that are yet to be identified.

$\mathrm{PINCH}$ is an independent prognostic factor in rectal cancer patients with RT, but not in patients without RT. The expression of PINCH in radiated colon cancer cells changed when analyzing expression in separately cultured to that in co-cultured cells, suggesting that the expression of PINCH may be regulated by radiation and by environmental factors surrounding the cancer cells. C-Myc significantly increased in co-cultured colon cancer cells with radiation compared to cell without radiation. In co-culture, C-Myc may be upregulated to protect cells from apoptosis induced by radiation. An in-depth cell line study and a study using a larger sample of rectal cancer patient material are ongoing to further confirm this relationship.

\section{Acknowledgements}

The authors are grateful to Dr Dianne Langford (Department of Neuroscience, Temple University School of Medicine, Philadelphia, PA, USA) who kindly provided us with the PINCH antibody. This study was supported by grants from the Foundation of Oncological Clinical Research in Linköping, the Swedish Cancer Foundation, Swedish Research Council and the Health Research Council in Southeast Sweden.

\section{References}

1. Rearden A: A new LIM protein containing an autoepitope homologous to 'senescent cell antigen'. Biochem Biophys Res Commun 201: 1124-1131, 1994.

2. Cabodi S, del Pilar Camacho-Leal M, Di Stefano P and Defilippi P: Integrin signalling adaptors: not only figurants in the cancer story. Nat Rev Cancer 10: 858-870, 2010.

3. Fukuda T, Chen K, Shi X and Wu C: PINCH-1 is an obligate partner of integrin-linked kinase (ILK) functioning in cell shape modulation, motility, and survival. J Biol Chem 278: 51324-51333, 2003.

4. Zhang Y, Chen K, Tu Y, et al: Assembly of the PINCH-ILKCH-ILKBP complex precedes and is essential for localization of each component to cell-matrix adhesion sites. J Cell Sci 115: 4777-4786, 2002.

5. Wu C: The PINCH-ILK-parvin complexes: assembly, functions and regulation. Biochim Biophys Acta 1692: 55-62, 2004.

6. Gao J, Arbman G, Rearden A and Sun XF: Stromal staining for PINCH is an independent prognostic indicator in colorectal cancer. Neoplasia 6: 796-801, 2004.

7. Holmqvist A, Gao J, Holmlund B, et al: PINCH is an independent prognostic factor in rectal cancer patients without preoperative radiotherapy - a study in a Swedish rectal cancer trial of preoperative radiotherapy. BMC Cancer 12: 65, 2012.

8. Eke I, Koch U, Hehlgans S, et al: PINCH1 regulates Akt1 activation and enhances radioresistance by inhibiting PP1alpha. J Clin Invest 120: 2516-2527, 2010.

9. Henriksson M and Luscher B: Proteins of the Myc network: essential regulators of cell growth and differentiation. Adv Cancer Res 68: 109-182, 1996.

10. Chen K, Tu Y, Zhang Y, Blair HC, Zhang L and Wu C: PINCH-1 regulates the ERK-Bim pathway and contributes to apoptosis resistance in cancer cells. J Biol Chem 283: 2508-2517, 2008.

11. Morikawa K, Walker SM, Nakajima M, Pathak S, Jessup JM and Fidler IJ: Influence of organ environment on the growth, selection, and metastasis of human colon carcinoma cells in nude mice. Cancer Res 48: 6863-6871, 1988.

12. Morikawa K, Walker SM, Jessup JM and Fidler IJ: In vivo selection of highly metastatic cells from surgical specimens of different primary human colon carcinomas implanted into nude mice. Cancer Res 48: 1943-1948, 1988.

13. No authors listed: Improved survival with preoperative radiotherapy in resectable rectal cancer. Swedish Rectal Cancer Trial. N Engl J Med 336: 980-987, 1997. 
14. Bustin SA, Benes V, Garson JA, et al: The MIQE guidelines: minimum information for publication of quantitative real-time PCR experiments. Clin Chem 55: 611-622, 2009.

15. Tu Y, Li F and Wu C: Nck-2, a novel Src homology2/3-containing adaptor protein that interacts with the LIM-only protein PINCH and components of growth factor receptor kinase-signaling pathways. Mol Biol Cell 9: 3367-3382, 1998.

16. Sandfort V, Eke I and Cordes N: The role of the focal adhesion protein PINCH1 for the radiosensitivity of adhesion and suspension cell cultures. PLoS One 5: pii: e13056, 2010.

17. Hague A,Hicks DJ, Bracey TS and Paraskeva C: Cell-cell contact and specific cytokines inhibit apoptosis of colonic epithelial cells: growth factors protect against c-myc-independent apoptosis. Br J Cancer 75: 960-968, 1997.

18. Ruifrok AC, Mason KA, Lozano G and Thames HD: Spatial and temporal patterns of expression of epidermal growth factor, transforming growth factor alpha and transforming growth factor beta 1-3 and their receptors in mouse jejunum after radiation treatment. Radiat Res 147: 1-12, 1997.
19. Schmidt-Ullrich RK, Valerie KC, Chan W and McWilliams D: Altered expression of epidermal growth factor receptor and estrogen receptor in MCF-7 cells after single and repeated radiation exposures. Int J Radiat Oncol Biol Phys 29: 813-819, 1994.

20. Greco C, Alvino S, Buglioni S, et al: Activation of c-MYC and c-MYB proto-oncogenes is associated with decreased apoptosis in tumor colon progression. Anticancer Res 21: 3185-3192, 2001.

21. Park EJ, Kiselev E, Conda-Sheridan M, Cushman M and Pezzuto JM: Induction of apoptosis by 3-amino-6-(3-aminopropyl)5,6-dihydro-5,11-dioxo-11H-indeno[1,2-c]isoquinoline via modulation of MAPKs (p38 and c-Jun N-terminal kinase) and c-Myc in HL-60 human leukemia cells. J Nat Prod 75: 378-384, 2012 . 